\title{
Measuring veterinary client preferences for autonomy and information when making medical decisions for their pets
}

\author{
Holli H. Seitz MPH, PhD \\ Jesse G. Grady DVM, MS
}

From the Department of Communication and Social Science Research Center (Seitz), and Department of Clinical Sciences, College of Veterinary Medicine (Grady), Mississippi State University, Mississippi State, MS 39762.

Address correspondence to Dr. Grady (jesse.grady@ msstate.edu).

\begin{abstract}
OBJECTIVE
To adapt the 3 scales of the Autonomy Preference Index to veterinary medicine and validate the 3 new scales to measure pet owner preferences for autonomy and information when making medical decisions for their pets.
\end{abstract}

\section{SAMPLE}

I0 small-animal veterinarians and 10 small-animal clients at a veterinary school-based community practice (pilot study) and $31 \mathrm{I}$ small-animal clients of the practice (validation study), of which 47 participated in a follow-up survey.

\section{PROCEDURES}

Wording of items in the Autonomy Preference Index was adapted, and instrument wording was finalized on the basis of feedback obtained in the pilot study to create 3 scales: the Veterinary General Decision-Making Preferences Scale (VGDMPS), Veterinary Clinical Decision-Making Preferences Scale (VCDMPS), and Veterinary Information-Seeking Preferences Scale (VISPS). The 3 scales were then validated by means of administering them to small-animal clients in a clinical setting.

\section{RESULTS}

The 3 scales had acceptable reliability and validity, but clients expressed concern over item wording in the VGDMPS during the pilot study. Overall, results showed that clients had a very high preference for information (mean \pm SD VISPS score, $4.78 \pm 0.36$ on a scale from I to 5 ). Preferences for autonomy varied, but mean values reflected a low-to-moderate desire for autonomy in clinical decision-making (mean \pm SD VCDMPS score, 2.04 \pm 0.62 on a scale from $I$ to 5 ).

\section{CONCLUSIONS AND CLINICAL RELEVANCE}

The VCDMPS was a reliable and valid instrument for measuring client preferences for autonomy in clinical decision-making. Veterinarians could potentially use this instrument to better understand pet owner preferences and tailor their communication approach accordingly.
$\mathbf{S}$ tudies examining pet owner adherence to recommendations for various elective and nonelective veterinary procedures have revealed variable adherence rates. A 2003 American Animal Hospital Association pet owner study $y^{1}$ found a $15 \%$ to $35 \%$ compliance rate for dental prophylaxis recommendations, dependent on dental disease grade; $18 \%$ and $19 \%$ compliance rates for cat and dog diet recommendations, respectively; and $32 \%$ and $35 \%$ compliance rates for screening laboratory testing for senior dogs and cats, respectively. Similarly, Kanji et $\mathrm{al}^{2}$ found that when receiving a recommendation for surgery, dentistry, or both, only $30 \%$ of pet owners complied. Both of these studies $^{1,2}$ found that pet owners' primary reasons for nonadherence were absent, inadequate, vague, or ambiguous recommendations. Furthermore, pet owners were 7 times as likely to adhere to recommendations if those recommendations were clear, and adherent clients had significantly higher satisfaction scores on post-visit surveys. ${ }^{2}$ One pet owner focus group study ${ }^{3}$ also found that pet owners expected veterinarians to educate them, provide choices, and use 2-way (interactive) communication to make a shared decision.
However, the amount of information individual pet owners desired as well as the degree of autonomy they required in making a shared decision varied. ${ }^{3}$

On the basis of these variable reported adherence rates, we theorized that veterinarians' perceptions of pet owners' desires for information, autonomy over their pet's health care, and desired method of receiving recommendations may be wrong. This supposition is supported by research showing that veterinarians' perceptions of their compliance rates have been overestimated, with, on average, a veterinarian with a $50 \%$ compliance rate assuming it was actually $75 \% .{ }^{1}$ Research has also shown that although veterinarians tend to convey information focusing on the value of their services, pet owners desire information that helps them understand their pets' health and wellbeing. ${ }^{2,3}$ And, although pet owners have expressed desire for information and options, the desired methods of receiving information and number of desired choices are not consistent among focus groups. ${ }^{2,3}$ With such variability in desires, how do veterinarians develop an effective way to communicate with individual pet owners? 
Despite evidence showing that pet owner preferences for shared decision-making vary, ${ }^{3}$ there is currently no instrument that assesses these preferences in veterinary medicine. However, several instruments do exist in human medicine. One such instrument in human medicine is the Autonomy Preference Index (API), a 23-item instrument that measures decision-making and information-seeking preferences. ${ }^{4}$ When administered to patients presenting to a general medicine practice, the API found that, in general, patients wanted to be informed but preferred that physicians make decisions, especially as the patient's illness severity increased. ${ }^{4}$ This widely adopted measure has been used to assess patient preferences for decision-making and information in surgical, primary care, and mental health settings as well as across general patient populations. ${ }^{5}$ Studies using the API have linked preferences for autonomy with patient satisfaction with the patient-doctor relationship. ${ }^{6}$ The API has also been used to show that as patient illness severity increases or patient health literacy decreases, patient participation in shared decision-making with their physician decreases. ${ }^{7,8}$

For the present study, we sought to adapt the API for veterinary medicine and validate this new instrument to measure pet owner preferences for autonomy and information in the context of making medical decisions for their pets. It is our hope that general practitioners and veterinary specialists will be able to use this instrument with their clientele to better understand pet owner desires for autonomy and information, effectively allowing them to tailor medical recommendations and information to individual pet owners.

\section{Materials and Methods}

The study protocol was approved by the Mississippi State University Institutional Review Board. All participants completed a consent form before participating in any phase of research.

\section{Pilot study}

We adapted the wording of items in the 3 scales of the $\mathrm{API}^{4}$ to be appropriate for veterinary medicine and created 3 vignettes that mirrored the type and severity of vignettes included in the API. Veterinarians and clients were then recruited to participate in nonincentivized, hour-long, semistructured, cognitiveprobing interviews to help improve the clarity and validity of the 3 adapted scales: the Veterinary General Decision-Making Preferences Scale (VGDMPS), Veterinary Clinical Decision-Making Preferences Scale (VCDMPS), and Veterinary Information-Seeking Preferences Scale (VISPS). A cognitive interview ${ }^{9}$ guide was developed to guide each interview. The interview guide for veterinarians included having them read each item of the adapted survey instrument, rephrase each statement in their own words, comment on how easy or difficult the item would be for their clients to answer, and make suggestions for improving the clarity of the item. For each situation in the vignette-based
VCDMPS, clinicians were also asked to rate the severity of each health issue, the realism of each scenario, and the medical accuracy of each scenario and the decisions that followed. The interview guide for clients was similar; clients read each statement, rephrased the statement in their own words, were asked to elaborate on any phrases that might be vague, discussed how they arrived at their answer, rated how easy or difficult it was to answer the item, rated the perceived realism of the scenarios, and made suggestions for clarifications.

Interviews were conducted from April 17, 2018, through May 18, 2018. A convenience sample of 10 small-animal veterinarians was recruited via a hospital-wide email from one of the authors (JGG) to veterinarians at the Mississippi State University College of Veterinary Medicine Animal Health Center (MSU-CVM AHC), a veterinary school-based smallanimal community practice in the southeastern US. A convenience sample of 10 small-animal veterinary clients were approached at the MSU-CVM AHC while waiting to see a community practice clinician. Participants who verbally agreed reviewed an electronic consent form and confirmed their willingness to participate. The online survey was presented to participants on a tablet computer. During the interviews, which were audio-recorded, participants read the survey on the tablet computer while responding to interviewer questions. Interviewers took notes on a printed interview guide.

After the first 6 interviews with veterinarians and 5 interviews with clients, we reviewed notes from the interviews and compiled a list of participant-proposed changes. Participants' rephrasing of items was compared with the intended meaning, and discrepancies were identified. We discussed all proposed changes and came to a consensus on how to revise survey items. Interviews resumed and were completed with an additional 4 veterinarians and 5 clients. After all interviews were completed, we again reviewed notes and discussed changes, and instrument wording was finalized on the basis of this additional feedback.

\section{Validation of scales with a veterinary hospital client population}

Procedure-Continuous in-hospital data collection began on July 26, 2018, and concluded on February 25,2019 . Potential pet owner participants were approached in the MSU-CVM AHC by one of the authors (JGG) while waiting to see a community practice clinician (with the exclusion of pet owners who had an appointment for euthanasia of their pet) and were invited to participate in an online survey. The online survey was presented to participants on a tablet computer, and participants who verbally agreed reviewed an electronic consent form and confirmed their willingness to participate. Participants who met screening criteria (ie, they had not previously participated in the survey, consented to participate, 
were $\geq 18$ years of age, and owned the pet they were with) completed the main survey while in the waiting room. On completion of the survey, participants were asked if they would be willing to participate in a follow-up survey via a final question in the main survey. Participants who completed the main survey were entered in a drawing to win 1 of $3 \$ 50$ electronic gift cards. Data collection ended when approximately 300 responses had been recorded.

An online follow-up survey was created to measure test-retest reliability of the scales. Follow-up data collection began on August 13, 2018, and concluded on October 8, 2018. With the goal of obtaining 50 follow-up responses, the first 91 participants who agreed to participate in the follow-up survey were contacted via email approximately 2 weeks after completing the first survey and were invited to complete the followup survey. Participants who completed the follow-up survey were sent a $\$ 5$ electronic gift card.

Instrument-The main survey instrument was an 85-item online survey administered via tablet computers in the MSU-CVM AHC. The survey included screening questions (age and pet ownership), reason for visit, the 6-item VGDMPS, the 9-item VCDMPS, the 8-item VISPS, 2 global measures of veterinary autonomy preference (adapted from measures used in human medicine), ${ }^{4,10}$ a global measure of pet attachment ${ }^{11}$ the 8 -item Companion Animal Bonding Scale (CABS), ${ }^{12}$ the 11 -item general attachment subscale of the Lexington Attachment to Pets Scale (LAPS), ${ }^{13}$ all 23 items from the API, ${ }^{4} 2$ global measures of patient autonomy used in human medicine,,${ }^{4,10}$ an item on the frequency of visits to the veterinarian, an item on the role of cost in decision-making about veterinary care, and demographic items (sex, marital status, race, ethnicity, education, employment, household size, and annual household income). All responses to items on the 3 adapted scales (VGDMPS, VCDMPS, and VISPS) were recoded such that higher scores reflected higher preferences for autonomy or information seeking; this included recoding reverse-scored items (4 items on the VGDMPS and 1 item on the VISPS).

The 25-item follow-up survey included all items from the VGDMPS, VCDMPS, and VISPS and the 2 global measures of patient autonomy presented in the main survey.

\section{Statistical analysis}

For each scale (VGDMPS, VCDMPS, and VISPS), we calculated the Cronbach $\alpha$ as a measure of internal consistency and as 1 measure of reliability. We also conducted principal component analysis with direct oblimin rotation to examine the factor structure of each scale, retaining factors with an eigenvalue $>1^{14}$ and interpreting factors with a loading $>0.4 . .^{15}$ After we determined which items to retain in each scale, each scale was scored by summing the scores for items in that scale and dividing by the number of items. We then calculated descriptive statistics (mean and SD) for individual items in each scale, for each scale, and for global measures included in the survey. Intraclass correlation coefficients (ICCs) were calculated for each scale to examine test-retest reliability. These ICCs were estimated, and their 95\% CIs were calculated on the basis of a mean rating $(\mathrm{k}=2)$, absolute agreement, 2-way, mixed-effects model. Correlations among scales (shortened VGDMPS and VISPS, VCDMPS, LAPS, and CABS) and global measures of autonomy were calculated with the Spearman method to assess convergent and discriminant validity. We then conducted an item response theory (IRT) analysis of each scale by means of graded response models. Finally, we conducted linear regressions to determine the relationship between participant characteristics and the scores for each scale. Specifically, we created 3 linear regression models for each of the three scales (VGDMPS, VCDMPS, and VISPS), in which the scale

Table I-Demographic characteristics of clients of a veterinary school-based small-animal community practice $(n=3 I I)$ who participated in a survey to validate 3 new scales developed to measure pet owner preferences for autonomy and information in the context of making medical decisions for their pets and of a subset of those clients (47) who participated in a follow-up survey.

\begin{tabular}{|c|c|c|}
\hline Demographic characteristic & $\begin{array}{l}\text { Main } \\
\text { survey }\end{array}$ & $\begin{array}{c}\text { Follow-up } \\
\text { survey }\end{array}$ \\
\hline Age (y) & $30.2(12.6)$ & $30.9(12.8)$ \\
\hline Female & 73.3 & 83.0 \\
\hline \multicolumn{3}{|l|}{ Marital status } \\
\hline Married & 32.8 & 34.0 \\
\hline Widowed & 0.6 & 0.0 \\
\hline Divorced & 2.6 & 4.3 \\
\hline Separated & 0.0 & 0.0 \\
\hline Never married & 64.0 & 61.7 \\
\hline \multicolumn{3}{|l|}{ Race* } \\
\hline White & 94.2 & 91.5 \\
\hline Black or African American & 3.5 & 0.0 \\
\hline American Indian or Alaska Native & 0.3 & 0.0 \\
\hline Asian & 2.9 & 4.3 \\
\hline Native Hawaiian or other Pacific Islander & 0.3 & 0.0 \\
\hline Other & 1.9 & 6.4 \\
\hline Hispanic, Latino, or Spanish origin & 5.5 & 6.4 \\
\hline \multicolumn{3}{|l|}{ Education } \\
\hline High school or less & 3.2 & 0.0 \\
\hline Attended college & 23.5 & 8.5 \\
\hline College graduate & 44.4 & 57.4 \\
\hline Advanced degree & 28.9 & 34.0 \\
\hline \multicolumn{3}{|l|}{ Employment } \\
\hline Unemployed & 4.2 & 8.5 \\
\hline Full-time student & 52.4 & 46.8 \\
\hline Part-time employed & 5.8 & 2.1 \\
\hline Full-time employed & 31.2 & 36.2 \\
\hline Military & 1.0 & 0.0 \\
\hline Legally disabled & 0.3 & 0.0 \\
\hline Retired & 5.1 & 6.4 \\
\hline \multicolumn{3}{|l|}{ Annual household income } \\
\hline$<\$ 20,000$ & 43.7 & 38.3 \\
\hline$\$ 20,000$ to $\$ 34,999$ & 13.6 & 21.3 \\
\hline$\$ 35,000$ to $\$ 54,999$ & 10.7 & 8.5 \\
\hline$\$ 55,000$ to $\$ 84,999$ & 9.1 & 10.6 \\
\hline$\geq \$ 85,000$ & 23.0 & 21.3 \\
\hline \multicolumn{3}{|l|}{ Pet owner* } \\
\hline Dog & 83.9 & 76.6 \\
\hline Cat & 48.4 & 51.1 \\
\hline
\end{tabular}

Data are given as percentages, except that age is given as mean (SD).

*Percentages may not total to 100 because participants could choose $>$ I response. 
Table 2-Mean item scores and SD for clients of a veterinary school-based small-animal community practice $(\mathrm{n}=3 \mathrm{II})$ who completed the Veterinary General Decision-Making Preferences Scale (VGDMPS) and item discrimination (a) and difficulty ( $b_{i}$ ) parameter values determined by means of item response theory (IRT).

\begin{tabular}{|c|c|c|c|c|c|c|c|}
\hline Item & $\begin{array}{l}\text { Mean (SD) } \\
\text { score* }\end{array}$ & a (SE) & $b_{1}$ (SE) & $b_{2}$ (SE) & $b_{3}(S E)$ & $b_{4}(\mathrm{SE})$ & $\begin{array}{l}\text { Cronbach } \alpha \\
\text { if item is } \\
\text { omitted }\end{array}$ \\
\hline $\begin{array}{l}\text { VGDMPSI: The important medical } \\
\text { decisions regarding your pet should be } \\
\text { made by your veterinarian, not by you. (R) }\end{array}$ & $2.59(1.21)$ & $2.57(0.4 I)$ & $-1.14(0.12)$ & $0.28(0.09)$ & $0.69(0.10)$ & $1.69(0.16)$ & 0.62 \\
\hline $\begin{array}{l}\text { VGDMPS2: You should go along with your } \\
\text { veterinarian's advice regarding your } \\
\text { pet even if you disagree with it. (R) }\end{array}$ & $2.90(1.21)$ & I.4I (0.18) & $-1.93(0.23)$ & $-0.13(0.11)$ & $0.40(0.11)$ & $2.14(0.25)$ & 0.65 \\
\hline $\begin{array}{l}\text { VGDMPS3: When your pet is hospitalized, } \\
\text { you should not be making decisions about } \\
\text { your pet's care. (R) }\end{array}$ & $3.36(\mathrm{I} .3 \mathrm{I})$ & $1.50(0.20)$ & $-1.96(0.23)$ & $-0.67(0.12)$ & $-0.26(0.11)$ & $1.17(0.16)$ & 0.63 \\
\hline $\begin{array}{l}\text { VGDMPS4: You should feel free to make } \\
\text { decisions about your pet's everyday } \\
\text { medical problems. }\end{array}$ & $4.08(0.97)$ & ND & ND & ND & ND & ND & 0.66 \\
\hline $\begin{array}{l}\text { VGDMPS5: If your pet were sick, as their } \\
\text { illness became worse, you would want } \\
\text { your veterinarian to take greater } \\
\text { control. (R) }\end{array}$ & $1.76(0.94)$ & $\mathrm{I} .80(0.26)$ & $-0.14(0.10)$ & $1.55(0.18)$ & $2.04(0.23)$ & $2.7 \mid(0.3 I)$ & 0.63 \\
\hline $\begin{array}{l}\text { VGDMPS6: You should decide how } \\
\text { frequently your pet needs a check-up. }\end{array}$ & 2.54 (I.18) & ND & ND & ND & ND & ND & 0.74 \\
\hline
\end{tabular}

Items followed by $(\mathrm{R})$ were intended to be reverse-scored, and data are given for the reversed version of that item. Cronbach $\alpha$ for the full scale $=0.70$. Cronbach $\alpha$ for the full scale when completed by a subset of clients $(n=47)$ at follow-up approximately 2 weeks later $=0.72$. Cronbach $\alpha$ for the 4-item scale (VGDMPSI, VGDMPS2, VGDMPS3, and VGDMPS5) $=0.74$. Cronbach $\alpha$ for the 4-item scale at follow-up $=0.75$.

$\mathrm{ND}=$ Not determined; discrimination $(a)$ and difficulty $\left(b_{i}\right)$ parameter values were not determined for these items because the Cronbach $\alpha$ increased with the omission of these items. $b_{1}=$ difficulty $\geq 2, b_{2}=$ difficulty $\geq 3, b_{3}=$ difficulty $\geq 4, b_{4}=$ difficulty $=5$.

*Items were scored on a scale from I to 5 , where I = strongly disagree, $2=$ somewhat disagree, $3=$ neither agree nor disagree, $4=$ somewhat agree, and $5=$ strongly agree.

score was used as the dependent variable and participant demographics and other characteristics were used as the predictor variables. Model 1 included participant age in years and gender; model 2 included age, gender, education in years, and annual income in thousands; and model 3 included age, gender, education, income, LAPS score, and CABS score.

\section{Results}

\section{Pilot study}

Of the 10 veterinarians who were interviewed, 3 were specialists and 7 were primary care providers. Their mean age was 35.2 years (SD, 10.8 years), and their mean years in practice was 10.4 years (SD, 9.3 years). Three were male, and 7 were female. Nine reported their race as white, and 1 reported their race as Black or African American. For the 10 clients who participated in interviews, mean age was 29.2 years (SD, 12.1 years). Three were male, and 7 were female. Eight reported their race as white, 1 as American Indian or Alaskan Native, and 1 as Asian. One participant reported being of Hispanic, Latino, or Spanish origin. Clients who agreed to be interviewed were well educated ( 1 attended college but did not graduate, 6 were college graduates, and 3 had graduate degrees), and the sample included both dog and cat owners.
For the VGDMPS, very few wording changes were suggested. However, some clients expressed surprise or verbally disagreed with the 4 reverse-scored items (ie, items that were worded in the direction of giving veterinarians control over decision-making). For example, in response to the item regarding decisions made when a pet is hospitalized, 1 client said, "It's the vet's job to guide you, ... but then it's still your pet, and the vet can't always make that decision."

For the VCDMPS, veterinarians and clients rated, on average, vignette 1 as least serious, vignette 2 as more serious, and vignette 3 as most serious. Veterinarians rated all vignettes as realistic and medically accurate. Clients also rated all vignettes as realistic. Interviews resulted in the addition of a definition for "heart murmur" in vignette 2 and in the clarification of language surrounding administration of pain medicine and decision-making regarding whether the pet should stay in the hospital or go home in vignette 3 .

For the VISPS, veterinarians and clients expressed confusion over the original wording of 2 items and suggested the addition of short clarifying phrases (ie, "You should be given information about your pet's bealth only when you ask for it" and "Information about your pet's illness is as important to you as information about the treatment"). Some par- 
Table 3-Mean item scores and SD for clients of a veterinary school-based small-animal community practice $(\mathrm{n}=3 \mathrm{II})$ who completed the Veterinary Clinical Decision-Making Preferences Scale (VCDMPS) and item discrimination (a) and difficulty $\left(\mathrm{b}_{\mathrm{i}}\right)$ parameter values determined by means of IRT.

\begin{tabular}{|c|c|c|c|c|c|c|c|}
\hline Item & $\begin{array}{c}\text { Mean (SD) } \\
\text { score* }\end{array}$ & a (SE) & $b_{1}$ (SE) & $b_{2}$ (SE) & $b_{3}(S E)$ & $\mathbf{b}_{4}$ (SE) & $\begin{array}{c}\text { Cronbach } \alpha \\
\text { if item } \\
\text { is omitted }\end{array}$ \\
\hline
\end{tabular}

Vignette I: Suppose your pet has had a runny nose and has been coughing for the last 3 days. You are about to call your veterinarian's office on the telephone. Who should make the following decisions?

VCDMPSI_I: Whether your pet should $2.88(0.99)$

be seen by the veterinarian.

VCDMPSI_2: Whether a chest $x$-ray should $2.15(0.84)$ be taken.

VCDMPSI_3: Whether your pet should $2.00(0.83)$

$0.84(0.13)-3.33(0.52)$

$-0.8 I(0.20) \quad I .7 I(0.28)$

$3.46(0.53)$

0.90

$2.09(0.22) \quad-0.93(0.11)$

$0.60(0.10)$

$2.30(0.22)$

$3.20(0.37)$

0.88

take medication.

Vignette 2: Suppose you took your pet to your veterinarian for a routine physical examination. The veterinarian found that your pet has developed a heart murmur (heart valve problem). Your pet has never had a heart murmur before. No other problems were found. Who should make the following decisions?

VCDMPS2_I: When the next visit to recheck the heart murmur should be. VCDMPS2_2: Whether a chest $\mathrm{x}$-ray

$$
1.91(0.83)
$$$$
1.72(0.19)-0.45(0.11)
$$

$0.93(0.12)$

$2.82(0.31)$

NA

$0.67(0.09)$

$2.42(0.22)$

$3.49(0.49)$

$0.64(0.09) \quad 2.39(0.22) \quad 3.36(0.46)$

$1.96(0.85) \quad 3.20(0.37) \quad-0.4$ I (0.08)

$0.64(0.09)$

be treated with medication.

Vignette 3: Suppose you witness your pet getting hit by a car. They are able to walk slowly but cry out when picked up. You rush them to the veterinarian. Who should make the following decisions?

VCDMPS3 I: Whether pain medication

should be given.

VCDMPS3_2: Whether x-rays should

I.8I (0.86)

$1.74(0.8 \mathrm{I})$

$2.16(0.24) \quad-0.17(0.09)$

$\mathrm{I} .0 \mathrm{I}(0 . \mathrm{II})$

$2.50(0.24)$

$3.42(0.43)$

$1.03(0.10)$

$2.46(0.23)$

$3.01(0.33)$

$1.97(0.85)$

$1.93(0.21) \quad-0.52(0.10)$

$0.79(0.11)$

$2.76(0.29)$

$3.53(0.46)$

0.88

Cronbach $\alpha$ for the full scale $=0.89$. Cronbach $\alpha$ for the full scale at follow-up $=0.90$. Cronbach $\alpha$ for vignette I $=0.68$. Cronbach $\alpha$ for vignette $2=0.85$. Cronbach $\alpha$ for vignette $3=0.86$

NA = Not applicable; a value could not be calculated because no respondents chose the corresponding response option.

*Items were scored on a scale from I to 5 , where I = the veterinarian alone, $2=$ mostly the veterinarian, $3=$ the veterinarian and you equally, $4=$ mostly you, and 5 = you alone. See Table 2 for remainder of key.

ticipants also verbally disagreed with or expressed confusion regarding the single item that was meant to be reverse-scored and thus worded in the opposite direction of other items in the scale.

\section{Validation of scales with a veterinary hospital client population}

Participants-A total of 374 clients were invited to participate in the study. Of these, 6 did not consent to participate, and 46 had already participated in the survey. Of the remaining 322 clients, 3 did not own the pet they had brought to the hospital, and their participation in the survey was terminated. Of the 319 clients who consented to participate and owned the pet they had brought to the visit, 311 (83.2\% of the original 374) completed the main survey. Mean age of participants was 30.2 years (SD, 12.6 years), and most participants were female (73.3\%), un-married (64.0\%), white (94.2\%), and non-Hispanic (94.5\%; Table I). Most had a college or graduate degree $(73.3 \%)$, approximately half were full-time students (52.4\%), and most had an income $<\$ 35,000(57.3 \%)$.

Overall, $78.5 \%$ of participants indicated a willingness to participate in the follow-up survey. Of the 91 participants invited to participate in the follow-up survey, 47 (51.6\%) completed it.
Internal consistency and principal component analysisFor the VGDMPS, internal consistency was highest when the 2 items that were not reverse-scored (VGDMPS 4 and VGDMPS6) were omitted, with the Cronbach $\alpha$ increasing from 0.70 to 0.74 with omission of these items (Table 2). The VCDMPS had high internal consistency without omitting any items (Cronbach $\alpha=0.89$; Table 3). For the VISPS, the internal consistency increased when the single reverse-scored item (VISPS5) was omitted, with the Cronbach $\alpha$ increasing from 0.65 to 0.80 with omission of this item (Table 4).

Principal component analysis suggested that the VGDMPS was composed of 2 factors, with the 4 reverse-scored items (VGDMPS1, VGDMPS2, VGDMPS3, and VGDMPS5) loading on 1 factor, 1 item (VGDMPS4) cross-loading on both factors, and the remaining item (VGDMPS6) loading on the second factor. Principal component analysis supported a single-factor solution for the VCDMPS but suggested a 2-factor solution for the VISPS scale, with the reverse-scored item (VISPS5) loading on the second factor and all other items loading on the first factor.

Descriptive statistics-Descriptive statistics were calculated for each item in the adapted scales (ie, after removal of 2 items from the VGDMPS and 1 item 
Table 4-Mean item scores and SD for clients of a veterinary school-based small-animal community practice $(n=3 \mathrm{I} I)$ who completed the Veterinary Information-Seeking Preferences Scale (VISPS) and item discrimination (a) and difficulty $\left(b_{i}\right)$ parameter values determined by means of IRT.

\begin{tabular}{|c|c|c|c|c|c|c|c|}
\hline Item & $\begin{array}{l}\text { Mean (SD) } \\
\text { score* }\end{array}$ & a (SE) & $b_{1}$ (SE) & $b_{2}(S E)$ & $b_{3}$ (SE) & $b_{4}$ (SE) & $\begin{array}{l}\text { Cronbach } \alpha \\
\text { if item } \\
\text { is omitted }\end{array}$ \\
\hline $\begin{array}{l}\text { VISPSI: As your pet becomes sicker, you } \\
\text { should be told more and more about your } \\
\text { pet's illness. }\end{array}$ & $4.74(0.62)$ & $1.29(0.23)$ & NA & $-4.31(0.75)$ & $-2.81(0.42)$ & $-1.46(0.21)$ & 0.61 \\
\hline $\begin{array}{l}\text { VISPS2: You should understand completely } \\
\text { what is happening inside your pet's body } \\
\text { as a result of their illness. }\end{array}$ & $4.49(0.8 I)$ & $1.66(0.26)$ & $-3.65(0.55)$ & $-2.58(0.32)$ & $-1.98(0.23)$ & $-0.46(0.10)$ & 0.60 \\
\hline $\begin{array}{l}\text { VISPS3: Even if the news about your pet's } \\
\text { condition is bad, you should be } \\
\text { well-informed. }\end{array}$ & $4.93(0.33)$ & $4.20(1.22)$ & NA & $-3.67(0.67)$ & $-2.85(0.39)$ & $-1.65(0.15)$ & 0.59 \\
\hline $\begin{array}{l}\text { VISPS4: Your veterinarian should explain } \\
\text { the purpose of your pet's laboratory tests. }\end{array}$ & $4.90(0.38)$ & $3.58(0.85)$ & NA & $-3.70(0.68)$ & $-2.67(0.33)$ & $-1.45(0.14)$ & 0.58 \\
\hline $\begin{array}{l}\text { VISPS5: You should be given information } \\
\text { about your pet's health only when you } \\
\text { ask for it. (R) }\end{array}$ & $4.17(1.2 I)$ & ND & ND & ND & ND & ND & 0.80 \\
\hline $\begin{array}{l}\text { VISPS6: It is important for you to know } \\
\text { all the side effects of your pet's } \\
\text { medication. }\end{array}$ & $4.76(0.53)$ & $1.77(0.30)$ & $-4.41(0.79)$ & $-3.50(0.51)$ & $-3.301(0.40)$ & $-1.09(0.14)$ & 0.59 \\
\hline $\begin{array}{l}\text { VISPS7: Information about your pet's illness } \\
\text { is as important to you as information } \\
\text { about the treatment. }\end{array}$ & $4.79(0.53)$ & $2.50(0.45)$ & $-3.87(0.70)$ & $-2.82(0.35)$ & $-2.49(0.28)$ & $-1.13(0.12)$ & 0.57 \\
\hline $\begin{array}{l}\text { VISPS8: When there is more than I method } \\
\text { to treat your pet's problem, you should } \\
\text { be told about each method. }\end{array}$ & $4.83(0.43)$ & $1.90(0.36)$ & NA & $-4.31(0.76)$ & $-3.65(0.58)$ & $-1.30(0.16)$ & 0.59 \\
\hline
\end{tabular}

Cronbach $\alpha$ for full scale $=0.65$. Cronbach $\alpha$ for full scale at follow-up $=0.64$. Cronbach $\alpha$ for 7 -item scale (VISPSI, VISPS2, VISPS3, VISPS4, VISPS6, VISPS7, and VISPS8) $=\alpha .80$. Cronbach $\alpha$ for 7 -item scale at follow-up $=0.79$.

*Items were scored on a scale from I to 5 , where I = strongly disagree, $2=$ somewhat disagree, 3 = neither agree nor disagree, 4 = somewhat agree, and 5 = strongly agree. See Tables 2 and 3 for remainder of key.

from the VISPS; Tables 2, 3, and 4). The mean client preference for autonomy in general decision-making contexts (ie, the 4-item VGDMPS) was 2.65 (SD, 0.88) on a scale of 1 to 5 , indicating a slight preference for the veterinarian to make the decision (Table 5). The mean client score for autonomy in clinical decisionmaking contexts (ie, the VCDMPS) was 2.04 (SD, 0.62 ) on a scale of 1 to 5 , which equated to the response that decisions should be made by "mostly the veterinarian." Within the VCDMPS, the mean client score for items in vignette 1 was 2.34 ( $\mathrm{SD}, 0.70)$, the mean client score for vignette 2 was 1.95 (SD, 0.73), and the mean client score for vignette 3 was 1.84 (SD, $0.74)$, all on a scale of 1 to 5 . The mean client score on the 7-item VISPS was $4.78(\mathrm{SD}, 0.36)$ on a scale of 1 to 5 , indicating a high preference for information.

Correlations between measures and test-retest reliability-The Spearman $\rho$ was calculated to assess correlations between adapted scales, adapted global measures of autonomy, and scales measuring pet attachment (Table 5). Both the VGDMPS and the VCDMPS were significantly and positively correlated with the global measures of preferences for control and decision-making. There was a small positive correlation between the
VGDMPS and the VISPS, but there was no correlation between the VCDMPS and the VISPS. There was a small positive correlation between the VISPS and the LAPS and between the VISPS and the CABS, a small negative correlation between the VCDMPS and the CABS, and no correlation between the VGDMPS and the LAPS or CABS. For each scale, we also calculated correlations between initial and follow-up results, and significant correlations were found for all 3 scales: 4-item VGDMPS $(\rho$ $=0.76 ; P<0.001)$, VCDMPS $(\rho=0.71 ; P<0.001)$, and 7 -item VISPS $(\rho=0.78 ; P<0.001)$. Intraclass correlation coefficients between initial and follow-up results were calculated to assess test-retest reliability. On the basis of standard interpretations that ICC values between 0.4 and 0.75 represent fair to good reliability and ICC values $>0.75$ represent excellent reliability, ${ }^{16}$ all 3 scales were considered to have acceptable test-retest reliability: 4-item VGDMPS (ICC $=0.847 ; 95 \% \mathrm{CI}, 0.726$ to 0.915 ), VCDMPS (ICC $=0.827 ; 95 \% \mathrm{CI}, 0.688$ to 0.903 ), and 7 -item VISPS (ICC $=0.854 ; 95 \%$ CI, 0.740 to 0.919 ).

\section{IRT analysis}

For all 3 scales, discrimination (a) and difficulty $\left(b_{i}\right)$ parameter values were calculated. Discrimina- 
Table 5-Mean (SD) scores for and correlations among various scales developed to measure pet owner preferences for autonomy and information in the context of making medical decisions for their pets and attachment to their pets.

Spearman $\rho$

\begin{tabular}{|c|c|c|c|c|c|c|c|}
\hline \multirow[b]{2}{*}{ Scale } & \\
\hline & $\begin{array}{l}\text { Mean }(S D) \\
\text { score }\end{array}$ & & 3 & 4 & 5 & 6 & 7 \\
\hline I. VGDMPS (4-item) & $2.65(0.88)$ & $0.504 *$ & $0.130 *$ & $0.519 *$ & $0.233 *$ & -0.018 & -0.038 \\
\hline 2. VCDMPS & $2.04(0.62)$ & & 0.035 & $0.531 *$ & $0.267 *$ & -0.098 & $-0.121 *$ \\
\hline 3. VISPS (7-item) & $4.78(0.36)$ & & & $0.149 *$ & $0.140 *$ & $0.17 \mid *$ & $0.156 *$ \\
\hline 4. Global measure of veterinary autonomy preference I & $2.81(0.70)$ & & & & $0.310 *$ & -0.005 & 0.033 \\
\hline 5. Global measure of veterinary autonomy preference 2 & $3.58(0.82)$ & & & & & 0.096 & 0.068 \\
\hline 6. LAPS (10-item) & $3.70(0.33)$ & & & & & & $0.494 *$ \\
\hline 7. CABS & $4.4 I(0.5 I)$ & & & & & & \\
\hline
\end{tabular}

For all scales, items were scored such that a higher score corresponded to a higher preference for autonomy, a higher preference for information, or a higher degree of attachment. Scores for the VGDMPS, VCDMPS, VISPS, global measure of veterinary autonomy preference I, global measure of veterinary autonomy preference 2, and CABS could range from I (low) to 5 (high). The I0-item LAPS refers to the general attachment subscale, excludes a reverse-scored item ("I am not very attached to my pet"), and is scored from I (low) to 4 (high).

$\mathrm{CABS}=$ Companion Animal Bonding Scale. LAPS $=$ Lexington Attachment to Pets Scale.

*Value was significantly $(P<0.05)$ different from 0 .

tion parameter values were interpreted as suggested by Baker. ${ }^{17}$ In short, items with higher discrimination parameter values were interpreted as being better able to distinguish between low and high levels of the underlying characteristic being measured than were items with lower discrimination parameter values.

Difficulty parameters, sometimes also referred to as location parameters, were interpreted as indicating the point (represented by $\theta$ ) at which a person, who possesses the underlying characteristic at the level of the parameter value, has a $50 \%$ chance of responding in a response category equal to or higher than that category.$^{18}$ For example, $a b_{1}$ value of -1.14 meant that participants with a $\theta$ equal to -1.14 (a preference below the midpoint of 0 ) had a $50 \%$ chance of answering 1 versus 2 or higher. Participants with a $\theta$ equal to 0.28 (preference just above the midpoint) had a 50\% chance of answering 1 or 2 versus 3 or higher.

Veterinary General Decision-Making Preferences Scale-The discrimination (a) parameter values were high or very high for the 4 items retained in the VGDMPS (Table 2 ). The difficulty $\left(b_{i}\right)$ parameter values for each item were relatively evenly spaced, indicating that the response categories allowed for differentiation among participants in measuring the characteristic.

Veterinary Clinical Decision-Making Preferences Scale-The discrimination (a) parameter values for items in the VCDMPS were all very high, with the exception of the value for 1 item (VDMPS1_1), which was moderate (Table 3 ). The difficulty $\left(b_{i}\right)$ parameter values for each item were relatively evenly spaced, indicating that the response categories allowed for differentiation among participants in measuring the characteristic.

Veterinary Information-Seeking Preferences ScaleThe discrimination (a) parameter values for the 7 items retained in the VISPS ranged from moderate (VISPS1) to high (VISPS2) to very high (VISPS3, VISPS4, VISPS6, VISPS7, and VISPS8). The difficulty $\left(b_{i}\right)$ parameter values were all negative, with values for some parameters missing because no participants selected response options corresponding to the lowest level of preference for information.

Differences by participant characteristics-Linear regression models were used to examine relationships between participant characteristics (age, gender, education, income, LAPS score, and CABS score) and scores for the VCDMPS and VISPS. Scores for the VCDMPS were not significantly associated with any of the participant characteristics we examined. Scores for the VISPS were positively related to the degree of pet attachment, as measured with the attachment subscale of the LAPS ${ }^{13}$ ( $b=0.26 ; P=0.001)$. This relationship was significant when controlling for age, gender, education, income, and companion animal bonding.

\section{Discussion}

To our knowledge, the present study was the first to propose and validate measures of veterinary client preference for autonomy in decision-making. Overall, results from interviews with veterinarians and clients suggested that scale items adapted from the $\mathrm{API}^{4}$ were acceptable in their accuracy and realism. The final versions of the 3 scales also had acceptable reliability (as determined on the basis of internal consistency and test-retest reliability) and validity (as determined by means of IRT analysis as 1 means of assessing construct validity and correlations with other measures to establish convergent and discriminant validity). Importantly, however, each scale had limitations.

Although the shortened form of the VGDMPS had acceptable internal consistency, test-retest reliability, convergent validity (correlation with global measures), and discriminant validity (no correlation with the VISPS, LAPS, or CABS) and the IRT analysis suggested that it could effectively differentiate clients with various degrees of preference for autonomy in general decision-making related to veterinary care of their pets, we do not recommend its use at this time. First, 2 of the 6 items were excluded to improve internal consistency and scale structure, but we sus- 
pect that their poor fit with the other items may have been due to the fact that the other items were reverse worded. ${ }^{19}$ Additionally, clients expressed concerns over the direction of the wording of the 4 retained items, in that the items suggested that veterinarians should be in control of decision-making (eg, "The important medical decisions regarding your pet should be made by your veterinarian, not by you"). Future research should examine the reliability and validity of an altered version of this instrument that takes those concerns into account, perhaps by reversing the wording of the 4 retained items so that all 6 items would be worded in the same direction and reflect higher client control. For example, the first item (VGDMPS1) could be revised to read "The important medical decisions regarding your pet should be made by you, not your veterinarian." Alternatively, it may be necessary to develop a new measure altogether that is not limited to items in the API.

The VCDMPS had acceptable internal consistency, test-retest reliability, convergent validity (correlation with global measures), and discriminant validity (no correlation with the LAPS or CABS) in its current form and did not elicit concerns from clients. Item response theory analysis suggested that the scale performed well in distinguishing between clients with low and high levels of preference for autonomy in clinical decision-making. The difficulty parameter values for the VCDMPS were slightly skewed (ie, most were $>$ the mean value of 0 ), thus these items were somewhat more useful in differentiating participants with a high desire for autonomy in clinical decision-making from participants with a low desire for autonomy. However, because many of these clinical items assessed decisions about tests and treatments, which veterinarians are responsible for recommending, many clients may not use the highest end of the scale for the VCDMPS. Indeed, the mean score for these items in our clinical population was approximately 2 , corresponding to a desire for decisions to be made by "mostly the veterinarian." Our findings also demonstrated that the desire for autonomy in clinical decision-making decreased as severity of the situation increased; this was consistent with findings in human medicine ${ }^{4}$ and suggested that veterinarians should consider not only typical individual preferences but also situational factors that might influence client preferences. The VCDMPS provided a tool for eliciting client preferences for autonomy in clinical decision-making and could potentially be administered to clients as a means for tailoring communication approaches. In human medicine, researchers have suggested that recognizing individual differences in autonomy preferences can enhance patient-centered care and improve patient outcomes. ${ }^{6}$ It is possible that improved veterinarian-client relationships could result from a similar emphasis on individual preferences in veterinary medicine. More research is needed to determine what communication approaches are most effective for clients at various levels of preference for autonomy and in different situations that vary in severity.
The shortened form of the VISPS had acceptable internal consistency, test-retest reliability, and discriminant validity (no correlation with the VGDMPS and only low correlations with the LAPS and CABS). These findings were similar to those in human medicine,${ }^{4}$ in which preferences for decision-making and information seeking are not highly correlated. Although we did not include a global measure of information-seeking preferences to assess convergent validity, the VISPS was significantly and positively correlated with the information-seeking preferences scale from the original API $(r=0.55 ; P<0.001)$. Participant preference for information, as measured by the VISPS, was very high, with few participants reporting a low desire for information. Because of this skewness in the data, estimated IRT parameter values may be biased because IRT analysis assumes that a latent characteristic has a normal distribution. ${ }^{20}$ Owing to the high mean reported levels of desire for information, the VISPS may not be useful in differentiating patients with differing needs and tailoring communication approaches. Rather, our data suggested that many clients prefer to feel well-informed. This has implications for how veterinarians present information. Finally, we do not fully understand how or whether information and autonomy preferences interact (ie, whether receiving more information would change an individual's preference for who makes a decision). Future research on measuring client preferences for information, tailoring information presentations to individual preferences, and examining the effects of information type and amount on preferences for autonomy in decision-making is needed.

In addition to validating the adapted scales, the present study allowed us to describe the autonomy and information preferences of a specific client population. Using the measures that were developed, we found that clients of the MSU-CVM AHC community practice had a low to moderate desire for autonomy in clinical decision-making and a high need for information. We also saw that as the severity of a pet's condition increased in the clinical setting (VCDMPS), a pet owner's desire for autonomy decreased. Our findings on information-seeking preferences were consistent with prior research by Coe et al, ${ }^{3}$ who found that pet owners who were not currently seeking veterinary care expected a high level of information from their veterinarians. These pet owners acknowledged that owners may vary in their desire for type and quantity of information, with other factors potentially affecting desired type and quantity. Uncertainty is one such factor that has been documented to increase a pet owner's motivation for information seeking, ${ }^{21}$ which likely explains why, despite our participants desiring less autonomy as their pet became more ill, they still desired a considerable amount of information.

The reason for visiting a veterinarian also likely affects the type but not necessarily the amount of information that pet owners desire. This has been demonstrated in a study of pet owners accessing 
tertiary oncology care for their pets who were more concerned about the truthfulness of the information than other information factors. ${ }^{22,23}$ Although the VISPS allows one to measure individual differences in preferences for information, pet owners did not vary as much as we expected. Future research may help veterinarians further define what factors most influence the type of information desired by pet owners, as research has found that physicians and veterinarians often incorrectly assume the type of information patients wish to hear. ${ }^{2,3,24}$ This in turn may help determine whether there are particular information factors that actually do influence decision-making, likely leading to better patient care and outcomes.

Because the present study was conducted at a veterinary school-based small-animal community practice, there were both strengths and limitations associated with the study population. On the one hand, measuring preferences for autonomy and information in a clinical setting was advantageous because it mimicked the setting in which clients might be responding to the final instrument. It also provided evidence of how the instrument would perform in a population of clients who were actively seeking veterinary care. On the other hand, responses might differ in nonclinical populations (ie, pet owners not seeking veterinary care at the time of surveying), in clinical populations in different geographic areas or at different types of veterinary practices (eg, private, referral, or corporate practices), or when administered by different veterinarians. Our study population was also overwhelmingly female at $73.3 \%$, which was much higher than the percentage of female pet owners (50.9\%) reported in the 2017-2018 AVMA Pet Ownership and Demographics Sourcebook. ${ }^{25}$ Future research should test the reliability and validity of the proposed scales in other populations with other demographic characteristics.

Also, our study population's socioeconomic makeup differed slightly from that for pet owners in general ${ }^{25}$ and substantially from that for the typical Mississippi resident. ${ }^{26}$ Most (73.3\%) of our participants held a college or advanced degree, whereas only $39.1 \%$ of pet owners and $21.3 \%$ of Mississippi residents hold a college degree. Most of our participants did, however, report an annual household income $<\$ 35,000$ (57.3\%), compared with $33 \%$ of pet owners in general and $42.7 \%$ of Mississippi residents who reported the same household income. It is likely that the reported income did not reflect the level of education of our participants because many participants were full-time students $(52.4 \%$, with some pursuing graduate study) and not employed full-time. Further research in different geographic and clinic settings will better elucidate any correlations between a pet owner's desire for autonomy and their socioeconomic status, income, or education.

In conclusion, the products of the present study were 2 -fold. First, we validated 2 scales to measure different aspects of pet owners' preferences for autonomy with regard to their pet's health care. Veterinar- ians can use the VCDMPS (clinical decision-making) and VISPS (information seeking) to better understand pet owner preferences and may be able to tailor their communication approach accordingly to better meet the needs of their clients and patients. It is our hope to further develop and simplify these instruments to allow veterinarians to use them in their practice, possibly by including them with new client intake forms. We also characterized the autonomy preferences (and the variables that influenced those preferences) within a population of clients at a veterinary schoolbased small-animal community practice. Preference for autonomy in decision-making was understandably lower in the clinical setting, where the veterinarian is responsible for recommending diagnostic tests and treatments, and decreased as the severity of the pet's condition increased. Finally, clients preferred a high degree of information, with those having higher pet attachment scores expressing a stronger preference for information. These results were similar to those reported in previously published focus group studies, ${ }^{3,27}$ but individual pet owner preferences may vary on the basis of other variables.

\section{Acknowledgments}

Funding was provided by the Stanton Foundation and the Mississippi State University College of Veterinary Medicine. Funding sources did not have any involvement in the study design, data analysis and interpretation, or writing and publication of the manuscript. The authors have no conflicts of interest to report.

\section{References}

1. The Path to High-Quality Care. Practical Tips for Improving Compliance. American Animal Hospital Association; 2003.

2. Kanji N, Coe JB, Adams CL, Shaw JR. Effect of veterinarianclient-patient interactions on client adherence to dentistry and surgery recommendations in companion-animal practice. J Am Vet Med Assoc. 2012;240(4):427-436.

3. Coe JB, Adams CL, Bonnett BN. A focus group study of veterinarians' and pet owners' perceptions of veterinarian-client communication in companion animal practice. J Am Vet Med Assoc. 2008;233(7):1072-1080.

4. Ende J, Kazis L, Ash A, Moskowitz MA. Measuring patients desire for autonomy: decision making and information-seeking preferences among medical patients. J Gen Intern Med. 1989;4(1):23-30.

5. Bonfils KA, Adams EL, Mueser KT, Wright-Berryman JL, Salyers MP. Factor structure of the autonomy preference index in people with severe mental illness. Psychiatry Res. 2015;228(3):526-530.

6. Lee Y-Y, Lin JL. Do patient autonomy preferences matter? Linking patient-centered care to patient-physician relationship and health outcomes. Soc Sci Med. 2010;71(10):1811-1818

7. Guadagnoli E, Ward P. Patient participation in decision-making. Soc Sci Med. 1998;47(3):329-339.

8. Longtin Y, Sax H, Leape LL, Sheridan SE, Donaldson L, Pittet D. Patient participation: current knowledge and applicability to patient safety. Mayo Clin Proc. 2010;85(1):53-62.

9. Willis GB, Artino AR Jr. What do our respondents think we're asking? Using cognitive interviewing to improve medical education surveys. J Grad Med Educ. 2013;5(3):353-356.

10. Sung VW, Raker CA, Myers DL, Clark MA. Treatment decisionmaking and information-seeking preferences in women with pelvic floor disorders. Int Urogynecol J. 2010;21(9):1071-1078.

11. AVMA. US Pet Ownership and Demographic Sourcebook. AVMA; 2012 
12. Poresky RH, Hendrix C, Mosier JE, Samuelson ML. The companion animal bonding scale: internal reliability and construct validity. Psychol Rep. 1987;60:743-746.

13. Johnson TP, Garrity TF, Stallones L. Psychometric evaluation of the Lexington Attachment to Pets Scale (LAPS). Antbrozoos. 1992;5(3):160-175.

14. Kaiser HF. The application of electronic computers to factor analysis. Educ Psychol Meas. 1960;20(1):141-151.

15. Pituch KA, Stevens J. Applied Multivariate Statistics for the Social Sciences: Analyses with SAS and IBM's SPSS. 6th ed. Routledge/Taylor \& Francis Group; 2016.

16. Fleiss JL. The Design and Analysis of Clinical Experiments. John Wiley \& Sons Inc; 1986.

17. Baker FB. The Basics of Item Response Theory. 2nd ed. ERIC Clearinghouse on Assessment and Evaluation; 2001.

18. Stata Item Response Theory Reference Manual: Release 16. Stata Press; 2019.

19. Zhang X, Noor R, Savalei V. Examining the effect of reverse worded items on the factor structure of the need for cognition scale. PLoS One. 2016;11(6):e0157795. doi: 10.1371/ journal.pone.0157795

20. Reise SP, Rodriguez A, Spritzer KL, Hays RD. Alternative approaches to addressing non-normal distributions in the application of IRT models to personality measures. J Pers Assess. 2018;100(4):363-374.
21. Stoewen DL, Coe JB, MacMartin C, Stone EA, Dewey CE. Identification of illness uncertainty in veterinary oncology: implications for service. Front Vet Sci. 2019;6:147. doi: 10.3389/fvets. 2019.00147

22. Stoewen DL, Coe JB, MacMartin C, Stone EA, Dewey CE. Qualitative study of the communication expectations of clients accessing oncology care at a tertiary referral center for dogs with life-limiting cancer. J Am Vet Med Assoc. 2014;245(7):785-795.

23. Stoewen DL, Coe JB, MacMartin C, Stone EA, Dewey CE. Qualitative study of the information expectations of clients accessing oncology care at a tertiary referral center for dogs with life-limiting cancer. $J$ Am Vet Med Assoc. 2014;245(7):773-783

24. Kindelan K, Kent G. Concordance between patients' information preferences and general practitioners' perceptions. Psychol Health. 1987;1(4):399-409.

25. AVMA. AVMA Pet Ownership and Demographics Sourcebook: 2017-2018 Edition. AVMA; 2018.

26. United States Census Bureau. American community survey. Accessed December 4, 2019. https://www.census.gov/acs/ www/data/data-tables-and-tools/data-profiles/

27. Coe JB, Adams CL, Bonnett BN. A focus group study of veterinarians' and pet owners' perceptions of the monetary aspects of veterinary care. J Am Vet Med Assoc. 2007;231(10):1510-1518. 